\title{
Reviews based on 1st Mediterranean Experts Meeting on the topic "Vitamin D in the prevention of health disparities during adult life"
}

\author{
Kalliopi Kotsa ${ }^{1}$. Spyridon Karras ${ }^{1}$ - Pantelis Zembekakis ${ }^{1}$
}

Published online: 2 March 2019

(C) Hellenic Endocrine Society 2019

Vitamin D has traditionally been considered a key player in skeletal health preservation [1]. During the past few decades, there has been increasing interest in vitamin D biology, as it has also been associated with the pathogenesis of several human diseases. Research in the vitamin D field, which is intense and booming, has established that this vitamin is actually a molecule that resembles a hormone more than a vitamin, with actions that extend well beyond its established musculoskeletal role. Its biological functions are thought to be exerted by interaction of the activated form 1,25(OH)2D (either D2 or D3) mainly with an intracellular receptor [2]. Most studies have focused on the association between 25-hydroxyvitamin $\mathrm{D}$ (as it is considered to best reflect vitamin D stores and availability), or the activated $1,25(\mathrm{OH}) 2 \mathrm{D}$, and disease processes. However, vitamin D biology is complex and, on the way to the formation of the final and prevailing metabolites, several intermediate molecules are generated that are no longer considered by-products or inactive [3]. Moreover, the "Mediterranean paradox" of vitamin D deficiency found to be prevalent in large parts of the population of countries with, nevertheless, abundant sunshine renders collaboration among scientists of these countries a necessity.

The need to join forces to understand the magnitude of the above phenomenon and the magnitude of the disorders

Kalliopi Kotsa

kalmanthou@yahoo.gr

1 Division of Endocrinology-Diabetes, 1st Dept. of Internal Medicine, Medical School, Aristotle University, Thessaloniki, Greece that may relate to this problem inspired us to set up a scientific workshop, named the Mediterranean Experts Meeting on Vitamin D, to date held two years running in Thessaloniki, Greece, to which we invited a large number of experts in the field who wished to contribute to vitamin D research by sharing knowledge, ideas, and proposals. Thus, two extremely successful Mediterranean Experts Meetings on "Vitamin D in the prevention of health disparities during adult life" were held in this Northern Greek city in 2017 and 2018. What started as an inspirational proposal among friends and colleagues became a friendly and highly professional exchange of scientific ideas and experience and has, meanwhile, evolved into a world-class scientific event (it will be hosted in Abu-Dhabi in 2019) thanks to the generous scientific input of all the distinguished participants.

This issue of Hormones gives its readers a brief overview of the scientific work presented mostly during the 2017 meeting. Professor Salvatore Minisola and colleagues deal with the issue of vitamin D supplementation [4], while Professors Erdinc Dursun and Duygu Gezen-Ak present their original work on vitamin $\mathrm{D}$ and neurodegeneration, with an emphasis on preclinical and clinical data on Alzheimer's disease [5, 6]. Professor William Grant scrutinizes the Mediterranean region, with particular focus on the potential association of vitamin D with disease status [7]. Finally, Maria Grammatiki, Spiros Karras, and Kalliopi Kotsa summarize preclinical and clinical data on vitamin $\mathrm{D}$ action on the pathogenesis of diabetes mellitus and complications [8].

By the end of both symposia, several important conclusions had been drawn on where we stand as regards the epidemiology and pathogenesis of vitamin D-related conditions as this pertains mostly to the Mediterranean region. 


\section{Compliance with ethical standards}

Conflict of interest The authors declare that they have no conflict of interest.

Publisher's note Springer Nature remains neutral with regard to jurisdictional claims in published maps and institutional affiliations.

\section{References}

1. HF DL (2008) Evolution of our understanding of vitamin D. Nutr Rev 66(10 Suppl 2):S73-S87

2. Holick MF (2008) The vitamin D deficiency pandemic and consequences for nonskeletal health: mechanisms of action. Mol Asp Med 29(6):361-368
3. Henry HL, Norman AW (1984) Vitamin D: metabolism and biological actions. Annu Rev Nutr 4:493-520

4. Salvatore Minisola et al (2018) Replenishment of vitamin D status: theoretical and practical considerations Hormones (Athens)

5. Erdinc Dursun, Duygu Gezen-Ak (2018) Vitamin D basis of Alzheimer's disease: from genetics to biomarkers Hormones (Athens)

6. Duygu Gezen-Ak, Erdinc Dursun (2018) Molecular basis of vitamin $\mathrm{D}$ action in neurodegeneration: the story of a team perspective Hormones (Athens)

7. William B. Grant (2018) Vitamin D and health in the Mediterranean countries Hormones (Athens)

8. Maria Grammatiki, Spiros Karras, Kalliopi Kotsa(2018) The role of vitamin $\mathrm{D}$ in the pathogenesis and treatment of diabetes mellitus: a narrative review Hormones (Athens) 